\title{
An optimized calibration strategy for high order adaptive optics systems : the Slope-Oriented Hadamard Actuation
}

\author{
Serge Meimon ${ }^{1, a}$, Thierry Fusco ${ }^{1}$, and Cyril Petit ${ }^{1}$ \\ Office National d'Études et de Recherches Aérospatiales \\ Département d'Optique Théorique et Appliquée \\ BP 72, F-92322 Châtillon cedex, France
}

\begin{abstract}
The correct calibration of the interaction matrix $D$ deeply affects the performance of an adaptive optics system. In the case of high-order systems, when the number of mirror modes is worth a few thousands, the actuation strategy is critical. This is a very first draft of a future paper, so feel free to contact the corresponding author for more accurate and up to date information.
\end{abstract}

\section{Introduction}

First, we propose a tractable interaction matrix quality criterion. Second, we derive its value in a calibration scheme context, and compare it to other interaction matrix calibration strategies(such as [1]) Last, we show simulation results in a SAXO-SPHERE case.

\section{Interaction matrix quality criterion}

Let $M_{\mathrm{inf}}$ be the influence function matrix - giving the modal decomposition of the shape of the mirror for a unitary displacement of each actuator - and $M_{\mathrm{wfs}}$ the wave front sensor (WFS) matrix - giving the WFS measurements for each phase mode. We suppose, with no lack of generality, that the phase modal basis size is very high, so the under-representation issues remain negligible.

The interaction matrix $D$ gives the WFS measurements for a unitary displacement of each actuator:

$$
D=M_{\mathrm{wfs}} \cdot M_{\mathrm{inf}} .
$$

Let $\hat{D}$ be the estimated interaction matrix. The true and estimated control matrices are given by:

$$
M_{\mathrm{com}} \stackrel{\Delta}{=} D^{\dagger}, \quad \hat{M}_{\mathrm{com}} \stackrel{\Delta}{=} \hat{D}^{\dagger}
$$

with $X^{\dagger}$ the pseudo-inverse of $X$.

The first issue we address is to define a $\hat{D}$ quality criterion, in order to compare various calibration strategies. To do so, we compute the residual phase and isolate the part due to miscalibration.

In a first step, we do not consider any dynamic(the impact of the control law will be discussed later): we put a known phase screen $\varphi_{\text {tur }}$ in front of the AO system, we compute the correction the system would apply $\varphi_{\text {cor }}$, and we call $\varphi_{\text {res }}$ the difqference. It is a "one-shot" use of the AO system.

$$
\varphi_{\mathrm{res}}(t)=\varphi_{\mathrm{tur}}(t)-\varphi_{\mathrm{cor}}(t)=\varphi_{\mathrm{tur}}(t)-M_{\mathrm{inf}} \cdot \hat{D}^{\dagger} \cdot\left[M_{\mathrm{wfs}} \cdot \varphi_{\mathrm{tur}}(t)+\text { noise }\right]
$$

This expression splits in the following terms:

$$
\varphi_{\mathrm{res}}(t)=\underbrace{\varphi_{\mathrm{tur}}(t)-M_{\mathrm{inf}} \cdot D^{\dagger} \cdot M_{\mathrm{wfs}} \cdot \varphi_{\mathrm{tur}}(t)}_{\text {fitting,aliasing }}-\underbrace{M_{\mathrm{inf}} \cdot D^{\dagger} \cdot \text { noise }}_{\text {WFS noise }}+\underbrace{M_{\mathrm{inf}} \cdot\left[D^{\dagger}-\hat{D}^{\dagger}\right] \cdot\left[M_{\mathrm{wfs}} \cdot \varphi_{\mathrm{tur}}(t)+\text { noise }\right]}_{\text {miscalibration }}
$$

\footnotetext{
a e-mail: authorname@onera.fr
} 
The miscalibration term contains a noise term and a turbulent term. For a WFS noise much lower than the signal $M_{\mathrm{wfs}} \cdot \varphi_{\mathrm{tur}}(t)$, the miscalibration term is approximated by:

$$
\varphi_{\mathrm{res}}^{\mathrm{cal}} \stackrel{\Delta}{=} M_{\mathrm{inf}} \cdot\left[D^{\dagger}-\hat{D}^{\dagger}\right] \cdot M_{\mathrm{wfs}} \cdot \varphi_{\mathrm{tur}}(t) .
$$

This term belongs to the image of $M_{\text {inf }}$, i.e. it is in the mirror space. We assume that all the mirror modes are seen by the WFS (or conversely that all the unseen mirror modes are filtered). Therefore, all of $\varphi_{\text {res }}^{\text {cal }}$ is seen by the WFS. In other words, the projection of $\varphi_{\text {res }}^{\text {cal }}$ onto the phase subspace observable by the WFS is $\varphi_{\mathrm{res}}^{\mathrm{cal}}$ itself. Mathematically, we define $\operatorname{Pr}^{\mathrm{obs}}=M_{\mathrm{wfs}}^{\dagger} \cdot M_{\mathrm{wfs}}$, which is the projector onto the WFS observable subspace, and write $\varphi_{\text {res }}^{\mathrm{cal}}=\operatorname{Pr}^{\mathrm{obs}} \varphi_{\mathrm{res}}^{\mathrm{cal}}$. With eqs. $(1,4)$, it comes:

$$
\varphi_{\mathrm{res}}^{\mathrm{cal}}=M_{\mathrm{wfs}}^{\dagger} D \cdot\left[D^{\dagger}-\hat{D}^{\dagger}\right] \cdot M_{\mathrm{wfs}} \cdot \varphi_{\mathrm{tur}}(t)
$$

We have $D \cdot\left[D^{\dagger}-\hat{D}^{\dagger}\right]=[\hat{D}-D] \cdot D^{\dagger}$ at the first order ${ }^{1}$,so

$$
\varphi_{\mathrm{res}}^{\mathrm{cal}}=M_{\mathrm{wfs}}^{\dagger}[\hat{D}-D] \cdot D^{\dagger} \cdot M_{\mathrm{wfs}} \cdot \varphi_{\mathrm{tur}}(t)
$$

An AO system is classically designed by optimizing the Strehl Ratio, or conversely by minimizing the temporal mean of the residual phase variance. Following this rationale, we define the interaction matrix quality by $Q(D) \stackrel{\Delta}{=}\left\langle\operatorname{Var}\left\{\varphi_{\mathrm{res}}^{\mathrm{cal}}\right\}\right\rangle_{t}$. We define $\Delta D \stackrel{\Delta}{=} \hat{D}-D$ and $C_{\text {slope }}=M_{\mathrm{wfs}} \cdot\left\langle\varphi_{\mathrm{tur}}(t) \cdot \varphi_{\mathrm{tur}}(t)^{\mathrm{T}}\right\rangle_{t} \cdot M_{\mathrm{wfs}}^{\dagger}$. With the norm defined on a vector or matrix

$$
\|M\|^{2}=\sum_{i, j}\left|M_{i, j}\right|^{2}=\operatorname{Trace}\left[M \cdot M^{\mathrm{T}}\right]=\operatorname{Trace}\left[M^{\mathrm{T}} \cdot M\right]
$$

and equation 6 , we obtain:

$$
Q(\Delta D)=\left\|M_{\mathrm{wfs}}^{\dagger} \cdot \Delta D \cdot D^{\dagger} \cdot \sqrt{C_{\text {slope }}}\right\|^{2}
$$

Of course, it is difficult to link this metric to the final contribution of the $D$ error in a closed loop configuration: the miscalibration error will result in an additionnal delay which depends on the selected control law. However, the tendancy will remain the same: two estimates of the calibration matrix with the same level of "static calibration error" $Q(\Delta D)$ will yield an equal delay in close loop (provided that the control law is linear). Therefore, the way to compare two strategies is the following: we compare the number of photons needed to reach the same level of static error for two calibration strategies. The best strategy is the less photon-consuming one.

\section{Interaction matrix calibration strategy}

In a calibration context, a set of actuation patterns $V_{i}$ is applied to the system. Let $V$ be the matrix which rows are the $V_{i}$. A classical way to proceed is to operate one actuator at a time, which corresponds to a diagonal matrix $V$. In what follows, we derive an optimized choice of $V$.

The set of measurements is $C=D V+N, N$ being the noise matrix, $N_{i, j}$ is the noise on the ith slope measurement for the jth pattern.. The estimated interaction matrix is $\hat{D}=C V^{\dagger}$. We gather

$$
\Delta D=-N V^{\dagger}
$$

Therefore, with equation 7 we get:

$$
Q(V)=\left\|M_{\mathrm{wfs}}^{\dagger} \cdot N V^{\dagger} \cdot D^{\dagger} \cdot \sqrt{C_{\text {slope }}}\right\|^{2}
$$

${ }^{1} D \cdot\left[D^{\dagger}-\hat{D}^{\dagger}\right]=[\hat{D}-D] \cdot \hat{D}^{\dagger} \simeq[\hat{D}-D] \cdot D^{\dagger}$. 
We assume that all the $N_{i, j}$ components are decorrelated, and that for a given subpupil, the noise variance should not change from one pattern to the other:

$$
\left\langle N_{i j} N_{k, l}\right\rangle=\delta_{i, k} \delta_{j, l} \sigma_{i}^{2}
$$

With this assumption, we can show that, in average,

$$
\langle Q(V)\rangle=\left\|M_{\mathrm{wfs}}^{\dagger} \cdot \Sigma_{N}\right\|^{2} \cdot\left\|V^{\dagger} \cdot D^{\dagger} \cdot \sqrt{C_{\text {slope }}}\right\|^{2}
$$

with $\Sigma_{N}=\operatorname{Diag}\left\{\sigma_{i}\right\}$. The optimum set of actuation patterns with respect to the residual phase variance is the one minimizing $\langle Q(V)\rangle$, or conversely, minimizing

$$
Q^{\prime}(V)=\left\|V^{\dagger} \cdot D^{\dagger} \cdot \sqrt{C_{\text {slope }}}\right\|^{2} .
$$

The minimization has to be done under some constraints:

Rank $V$ must be of full rank, otherwise $V^{\dagger}$ has no meaning;

DMsat $\left|V_{i}\right| \leq V_{\max }, V_{\max }$ being the saturation level of the DM;

WFSsat $\left|[D \cdot V]_{i}\right| \leq S_{\max }, S_{\max }$ being the limit of the linearity domain of the WFS.

\section{Optimization methods}

In the general case, this optimization problem is not easy, and we have not found a full optimization method. However, under some asumptions, the solution is straightforward.

Voltage Oriented Hadamard Actuation For instance, we consider the approximation

$$
D^{\dagger} \cdot \sqrt{C_{\text {slope }}} \simeq \mathbf{I d},
$$

and restrain the choice of $V$ to invertible matrices (i.e. a set of $N_{\text {actindependant patterns), so that }}$ $V^{\dagger}=V^{-1}$ ). Taking into account only the constraints Rank and DMsat, the optimization problem reads:

$$
V_{\text {opt }}=\underset{\left|V_{i}\right| \leq V_{\max }}{\arg \min }\left\|V^{\dagger}\right\|^{2}=\underset{\left|V_{i}\right| \leq V_{\max }, \operatorname{det}(V) \neq 0}{\arg \max }\|V\|^{2}
$$

Let $N_{\text {act }}$ be the number of actuators. So $V_{\text {opt }}$ contains $N_{\text {act }}^{2}$ components lower than $V_{\max }$ in absolute value. Then $\left\|V_{\text {opt }}\right\|^{2} \leq N_{\text {act }}^{2} V_{\max }$. The Hadamard matrices $H_{n}$ are invertible (and orthogonal) matrices containing \pm 1 components. The matrix $V_{\max } \cdot H_{N_{\text {act }}}$ has a norm of $N_{\text {act }}^{2} V_{\max }$ and is invertible. It is therefore a solution to the optimization problem 12:

$$
V_{\mathrm{opt}}=V_{\max } \cdot H_{N_{\mathrm{act}}}
$$

This solution has been proposed and qualified by [[1]], although it was obtained with a different approach.

Slope Oriented Hadamard Actuation First, the approximation 11 is questionable: it implies that statistically, all actuators displacements are decorelated and have the same energy. Simulation on a SAXO case (with a $r_{0}$ of $18 \mathrm{~cm}$ at 0.7 microns) show that it is not the case (see fig. 1). However, the approximation

$$
\sqrt{C_{\text {slope }}} \simeq \mathbf{I d}
$$




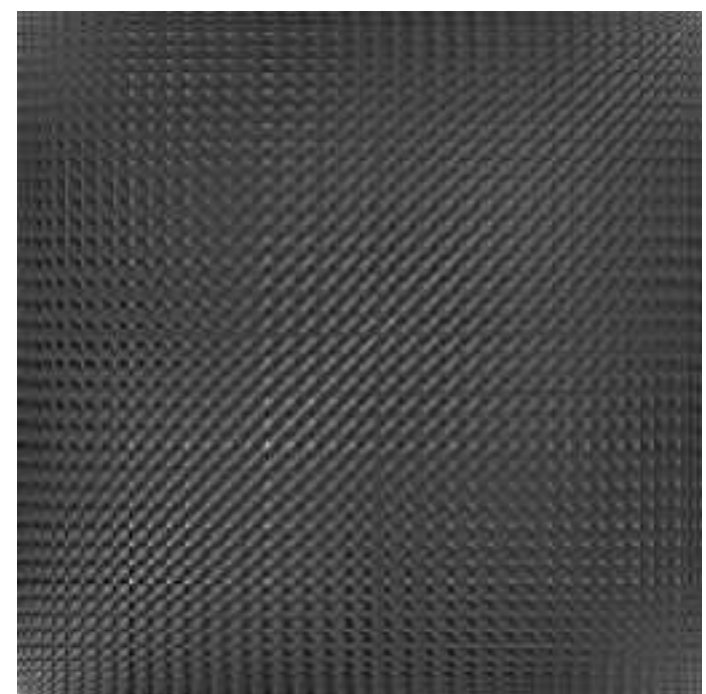

Fig. 1. Actuator voltages covariance matrix

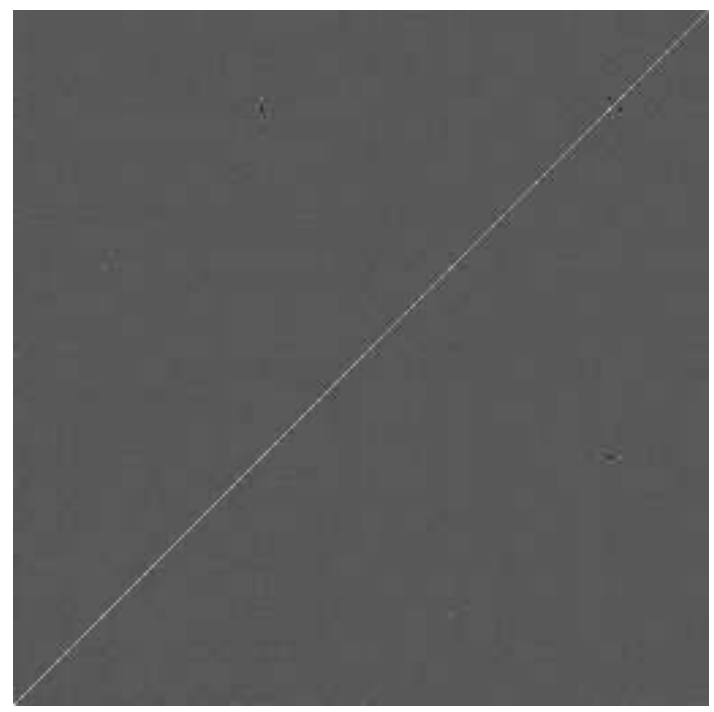

Fig. 2. Slopes covariance matrix

is valid at the first order $^{2}$ (see fig. 2).

Second, the constraint we have to keep is WFSsat, and not DMsat: the DM is designed to compensate for the turbulence whereas the WFS is designed to measure the residual turbulence. In a calibration scheme, the saturation limit of the WFS will be met much sooner than the DM saturation limit. The optimization problem then reads:

$$
V_{\mathrm{opt}}=\underset{\left|[D V]_{i}\right| \leq S_{\max }}{\arg \min }\left\|V^{\dagger} D^{\dagger}\right\|^{2}
$$

Although this optimization problem is not straightforward to solve, there exists a degraded solution directly computable. Let us suppose that both $D$ and $V$ are invertible. Then the optimization problem

\footnotetext{
2 if we neglect the cross correlation of $\mathrm{x} / \mathrm{y}$ slopes and the cross-correlation between sub-pertures distant of less than $r_{0}$ tipically
} 
reads:

$$
V_{\text {opt }}=\underset{\left|[D V]_{i}\right| \leq S_{\max }, \operatorname{det}(D V) \neq 0}{\arg \max }\|D V\|^{2}
$$

which has the same shape as 12 in the slope space. The obtained solution then verifies $D V_{\text {opt }}=S_{\max }$. $H_{N_{\text {slope }}}$, so

$$
V_{\mathrm{opt}}=S_{\max } \cdot D^{\dagger} H_{N_{\mathrm{act}}}
$$

Here, the idea is to find the actuation pattern such that all the WFS slopes are at the saturation limit. It will yield the best SNR possible.

There are two drawbacks for this solution:

- it requires the knowledge of $D^{\dagger}$ to be computed. In practice, a first rough estimate of $D$ (with a high noise, or a synthetic matrix) can be used to compute $V_{\text {opt }}$. This $V_{\text {opt }}$ is then used in a second step to perform a fine calibration;

- it is not an exact solution. Therefore, it might saturate the WFS : nothing ensures that $D \cdot V_{\text {opt }}=$ $S_{\max } \cdot D \cdot D^{\dagger} H_{N_{\text {act }}}$ has components lower that $S_{\max }$ in absolute value. However, the saturation level of a WFS is seldom a hard limit. Anyway, a tuning of $S_{\max }$ so that the WFS is not saturated is required. Actually, the voltage oriented Hadamard Actuation also requires some tuning to avoid WFS saturation, as proposed by [[1]], so this argument cannot be used to select one method or the other.

\section{Simulation results}

The total error on the interaction matrix contains a noise error and a linearity error: for high voltages, the SNR is better and the noise propagation is low, but the WFS saturation error is higher. To compare calibration strategies, one must tune the overall voltage gain so that the saturation error (i.e. the calibration error with no noise) is the same. Then, the strategy corresponding to the lowest noise propagation is the winner. We have performed a diffractive simulation of the calibration proceedure in a SAXO case (40x40 subpupils, $6 \times 6$ pixels per sub pupil, pinhole diameter worth a $50^{\text {th }}$ of the full pupil). We have assumed a uniform slope noise over all the subpupils. We have considered three strategies:

IF the influence function method, corresponding to sequential measurement for each actuator; HA the Hadamard Actuation method[1];

SOHA the Slope Oriented Hadamard Actuation method, wich we previously described.

Overall voltage gains have been tuned so that all three yield the same saturation error.

We have previously shown that the approximation 14 is valid. With this, the interaction matrix error metric of eq. 7 becomes

$$
Q(\Delta D)=\left\|M_{\mathrm{wfs}}^{\dagger} \cdot \Delta D \cdot D^{\dagger}\right\|^{2}
$$

. As previously mentionned, this quality criterion has no absolute meaning in a close loop operation. To give it more meaning, we normalize it by $Q(D)$, which is the error obtained with a null estimation $(\hat{D}=0)$ :

$$
\bar{Q} \stackrel{\Delta}{=} \frac{Q(\Delta D)}{Q(D)}
$$

It is equal to $100 \%$ if the error is $100 \%$ of the matrix itself. This is the metric used in fig 3 . The rough estimate of $D$ used for the SOHA method had a relative error slightly below $50 \%$ (dotted line).

The way to use these results is to compare the time (or photons) necessary for each method to reach one given quality of $D$ estimation:

- the gain from IF to HA method is a 100 factor gain in calibration time in photon noise regime (or 6.3 magnitudes);

- the gain from HA to SOHA method is a 10 factor gain in calibration time in photon noise regime (or 2.1 magnitudes). 
First conference on Adaptive Optics for Extremely Large Telescopes

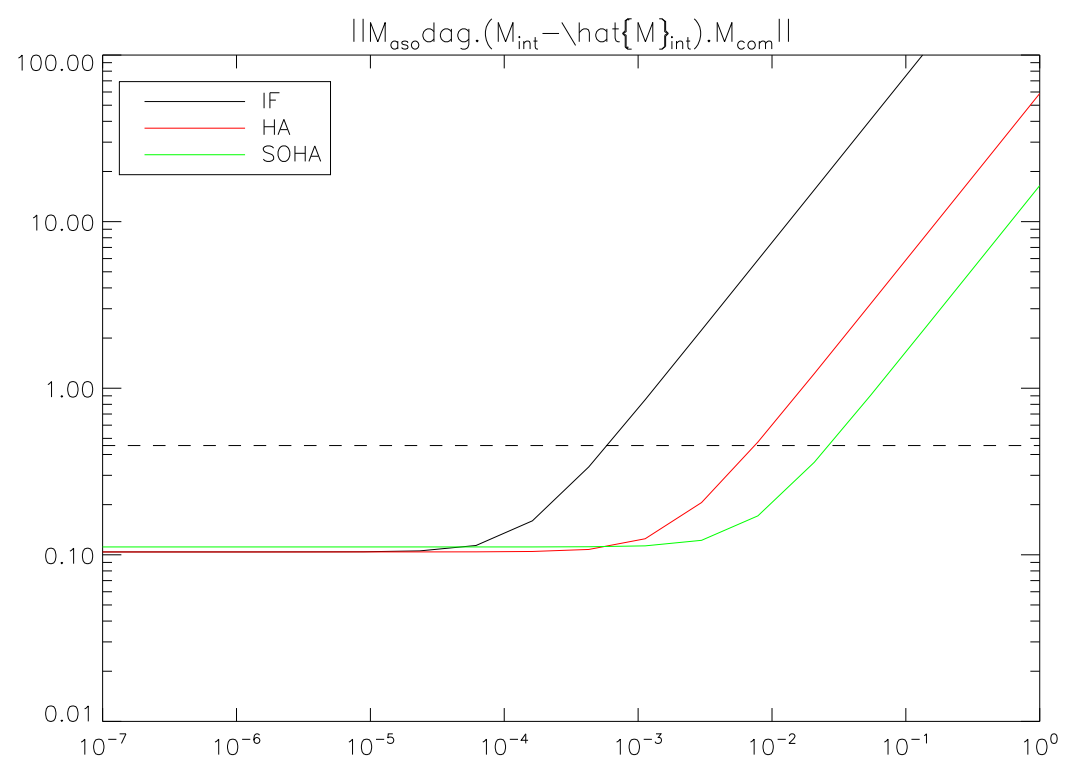

Fig. 3. Interaction matrix quality $(\bar{Q})$ in function of the slope measurement error (in radians edge to edge).

\section{References}

1. M. Kasper, E. Fedrigo, D. P. Looze, H. Bonnet, I. L., and S. Oberti, "Fast calibration of high-order adaptive optics systems," J. Opt. Soc. Am. A 21 (2004). 